\title{
Is 2-step test useful for screening for cognitive decline?
}

\author{
Abstract \\ Purpose: Cognitive decline like "mild cognitive impairment (MCI)" participants are \\ describing have a locomotive dysfunction as a low walking speed (WS). The purpose is \\ verifying that 2-step test is useful screening for MCI. \\ Methods: One hundred seventy seven participants were recruited and classified into MCI \\ and non-MCI by the rapid dementia screening test-Japanese. Evaluation was used walking \\ speed (normal and maximum) and 2-step test for participants. \\ Results: Thirty seven MCI participants had slow WS and short 2-steps length. However \\ in adjusted by age and education years, only 2-step test was significantly lower in MCI. \\ Conclusion: Two-step test might be useful for screening for MCI better than WS. \\ Keywords: Cognitive decline, Mild cognitive impairment, screening, Locomotive \\ function, 2-step test, Elderly people
}

Volume 7 Issue 5 - 2017

\author{
Kohei Maruya,' Hiroaki Fujita,' Tomoyuki \\ Arai,' Kenichi Ogiwara, ${ }^{2}$ Syunichiro \\ Moriyama ${ }^{2}$ \\ 'Department of Health and Medical Care, Saitama Medica \\ University, Japan \\ ${ }^{2}$ Department of Rehabilitation, Musashidai Hospital, Japan
}

Correspondence: Kohei Maruya, Faculty of health and Medical Care, Saitama Medical University, Moroyama town, Saitama prefecture, Japan, Tel 8|49-295-1001, Fax 8|49-295-5104, Emailmaruya@saitama-med.ac.jp

Received: February 24, 2017 | Published: March 20, 2017
Abbreviations: MCI, Mild Cognitive Impairment; WS, Walking Speed; RDST-J, The Rapid Dementia Screening Test Japanese

\section{Introduction}

Recently, cognitive decline like "mild cognitive impairment (MCI)" is reported that step length and Walking speed (WS) are decreased. ${ }^{1}$ However indication of low WS is described it is under $0.8 \square 1.0 \mathrm{~m} / \mathrm{s}$. These WS are suspected that subjects are already frailty. MCI lurk and the symptom usually would not appear in the activity daily living. It is important to prevent dementia as soon as possible. Therefore tool is necessity for available to detect earlier. 2-step test is correlated with WS and can be screen for locomotive dysfunction. ${ }^{2}$ In this study, purpose is validation of screening for MCI by 2-step test.

\section{Methods}

One hundred seventy seven participants who lived independent in over 65 years old were recruited in a health education seminar.
In this study, MCI was judged by the rapid dementia screening test - Japanese (RDST-J), with MCI below 7 point. $^{3}$ The assessment of locomotive function was used for WS (normal and maximum) and 2-step test. Participants were asked the number of years of education as confounding factor. WS was measured in 6-meter length walking road with comfortable speed in usual and maximum speed in possible effort. 2-step test was measured maximum of two steps length. Moreover 2-step value was calculated that two steps length divided by height. The study was approved by ethics board of the faculty of health and medical care of Saitama medical university (No.137), and participants provided informed consent.

\section{Analysis}

Participants were divided MCI and non-MCI by RDST-J. WS and 2-step value were compared MCI to non-MCI by t-test. In addition, adjusted t-test to age and a number of educations was carried out. All statistical analyzes were performed using JMP (version 11.0 for Mac; SAS), with a $\mathrm{P}<0.05$ considered as the level of significance.

Table I Compared of characteristics between $\mathrm{MCl}$ and non-MCI

\begin{tabular}{|c|c|c|c|c|c|c|}
\hline \multicolumn{7}{|l|}{ Unadjust } \\
\hline & Age,Year & Femail, n (\%)§ & Education years, year & normal WS, $\mathrm{m} / \mathrm{s}$ & maximal WS, m/s & 2-step value, $\mathrm{m} / \mathrm{m}$ \\
\hline Overall $(n=177)$ & $73.9(5.7)$ & $|5|(85.3)$ & $11.9(2.0)$ & $1.36(0.24)$ & $\mathrm{I} .82(0.33)$ & $1.38(0.18)$ \\
\hline $\mathrm{MCl}(\mathrm{n}=37)$ & $78.1(0.87)$ & $29(78.4)$ & $12.0(1.9)$ & $1.24(0.25)$ & $1.65(0.30)$ & $1.26(0.16)$ \\
\hline non-MCl $(n=140)$ & $72.9(0.45)$ & $122(87.1)$ & $11.9(2.0)$ & $1.39(0.23)$ & $1.86(0.32)$ & $1.42(0.17)$ \\
\hline p-value & $<0.0001$ & 0.1956 & 0.7234 & 0.0005 & 0.0005 & $<0.0001$ \\
\hline \multicolumn{7}{|c|}{ Adjusted by age $\&$ education years } \\
\hline & Age,Year & Female, n (\%) & Education Years, year & Normal WS, m/s & Maximal WS, $\mathrm{m} / \mathrm{s}$ & 2-step Value, $\mathrm{m} / \mathrm{m}$ \\
\hline $\mathrm{MCl}(\mathrm{n}=37)$ & - & - & - & I.3। (0.04) & $1.75(0.06)$ & $1.32(0.03)$ \\
\hline non-MCl $(n=\mid 40)$ & - & - & - & $\mathrm{I} .40(0.02)$ & $1.85(0.03)$ & $1.39(0.01)$ \\
\hline $\mathrm{p}$-value & - & - & - & 0.0843 & 0.1099 & 0.0453 \\
\hline
\end{tabular}

Unadjust: Mean (standard deviation);Ajusted: Estimate (standard error); t-test; §: chi-square test MCI: Mild Cognitive Impairment;WS:Walking Speed.

\section{Results}

Thirty seven participants $(20.9 \%)$ were MCI in all participants. Average age of MCI was significantly higher than non-MCI (78.9 vs 72.9 years). No significant was sex and a number of education. Normal and maximum WS were significantly lower in MCI participants. The 2-step value was significant low in MCI. However, only 2-step value in MCI was significant low in adjusted by age and number of education analysis (Table 1). 


\section{Discussion}

In this study, 37 participants were judged MCI. They were older than non-MCI and had lower WS and 2-step value. However, the walking speed was higher than previous reports. In other words, it is considered that there is possible that cognitive decline is appeared before physical function is weakened. Furthermore in compare in MCI and non-MCI adjusted by age and education years, 2-step value was only significant. It is suspected that 2-step value is decreased along with cognitive function irrespective of age and education years. Therefore 2-step test might be useful for screening for MCI. However it does not ravel the causal relationship between MCI and 2-step test because this study is a cross-sectional study. We need further research and more participants.

\section{Conclusion}

The poor results of the 2-step test could suggest locomotive dysfunction and cognitive decline irrespective of age and education years in the elderly. Therefore, the 2-step test might be useful for MCI screening.

\section{Acknowledgements}

Thank you for joining all staff and participants in this study.

\section{Conflicts of interest}

None.

\section{References}

1. Verghese J, Robbins M, Holtzer R, et al. Gait dysfunction in mild cognitive impairment syndromes. J Am Geriatr Soc. 2008;56(7):1244-1251.

2. Muranaga S, Harano K. Development of a convenient way to predict ability to walk, using a two-step test. J Showa Med Associat. 2003;63(3):301-308.

3. Sakai Y. Japanese version of the rapid dementia screening test: Effectiveness in detecting possible patients with dementia. Jpn J Geriatr Psychiatry. 2006;17(5):539-551. 